\title{
SCIDiC
}

\author{
International Journal of Dentistry and Oral Science (IJDOS) \\ ISSN: 2377-8075
}

\section{Knowledge and Attitude of Dental Interns about Management of Tooth Avulsion: A Comparative Cross - Sectional Study}

Research Article

Rabaa M. Aboubakr ${ }^{1}$, Wahdan M.A. Elkwatehy ${ }^{2 *}$

Assistant Professor of Pediatric \& Dental Public Health and Preventive Dentistry, Mansoura University, Egypt.

\section{Abstract}

Objectives: This study aimed to assess the knowledge and attitude of Saudi and Egyptian dental interns about management of avulsed teeth and to compare between them.

Methods: This cross sectional study was conducted during the period from October, 2019 till December, 2019 after obtaining ethical approval from the institutional research committee in Faculty of Dentistry, Mansoura University. A well-designed questionnaire consists of two parts; part I about demographic data and part II about knowledge of interns was distributed. Data from questionnaires was collected and analyzed using SPSS version 21.0. Chi square test was used to assess percentage and frequencies of independent variables and the relation between the knowledge and the independent variables was estimated.

Results: 324 dental interns out of 415 with response rate $78 \%$ participated in the study and returned the completed questionnaires on the same day. Dental interns with more than 6 months' experience and prior knowledge about management of avulsed tooth were better in answering the questions. Dental interns who had not attended an educational program regarding traumatic injuries to teeth showed significant incorrect answers. There were no significant differences between Saudi and Egyptian dental interns in answering 5 from 8 questions related to knowledge about avulsion tooth. Also, there were no significant differences between male and female dental interns who answering 6 from 8 questions correctly.

Conclusion: The Saudi and Egyptian dental interns had sufficient level of knowledge about most items regarding tooth avulsion management.

\section{Introduction}

Avulsion is defined as a condition where tooth is completely lost from its socket. It is known as the most serious form of Traumatic Dental Injuries (TDIs) and represents 1\%-16\% of all dental injuries $[1,2]$. Permanent dentition is affected by TDIs more than primary dentition (58.6\% vs $36.8 \%$ respectively), and the maxillary anterior teeth are the most affected [3]. Most cases with tooth avulsion occur between ages 8 and 11 years $[4,5]$.

Tooth avulsion management depends on the dentition's type. Replantation of primary teeth is not recommended as it may affect their permanent successors, and the only treatment option is clinical and radiographic follow up of these teeth until eruption of their permanent successors $[4,5]$. However, management of permanent tooth avulsion has different protocol as the tooth should be handled from the crown, and if it is contaminated, it must be cleaned using normal saline or cold running water before reposi- tioning, after that the avulsed tooth is repositioned, and patient should bite gently on a handkerchief to secure it in its socket, if repositioning was not possible, the avulsed tooth must be stored in a physiological medium such as milk, saliva, or saline. Tooth should not be stored in water. Finally, the patient must visit the dentist immediately [6].

It is known that, the main factors that affect success rate of tooth replantation are; factors which directly affect the viability of periodontal ligament cells such as transportation method and media and time period takes place outside the socket [7]. The American Academy of Pediatric Dentistry (AAPD) and the International Association for Dental Traumatology (IADT), recommended immediate replantation of an avulsed tooth to get better prognosis $[2,8]$. Previous studies denoted that, tooth should be stored in a storage media if it cannot be replanted within 5 minutes [9-11] as the risk of ankylosis after replantation of avulsed tooth increased with increasing the time of dryness over 20 min [12-14].

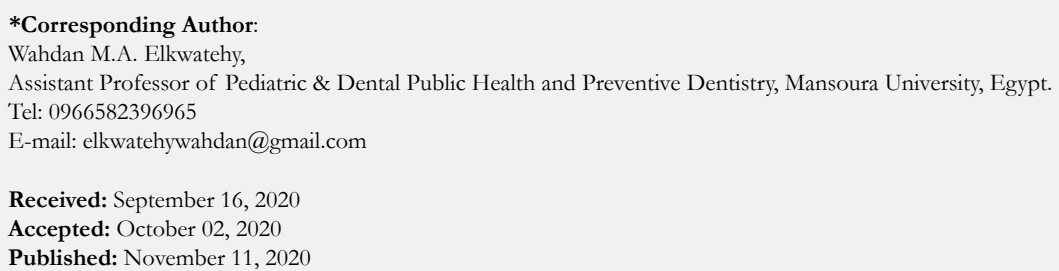

Copyright: Wahdan M.A. Elkwatehy ${ }^{\circ} 020$. This is an open-access article distributed under the terms of the Creative Commons Attribution License, which permits unrestricted use, distribution and reproduction in any medium, provided the original author and source are credited. 
The incidence of dental trauma in Saudi Arabia is higher than that of other countries $[15,16]$. Regarding the prevalence of TDI in Saudi Arabia, it was 34\% among 12-14 years old [16]. However, in Egypt it was reported to be $14.6 \%$ among $8-12$ years old children [17], and $13.6 \%$ among $11-14$ years [18].

Dental trauma should be taught as a first aid condition and an emergency treatment is needed as early as possible. Peoples consider interns and newly undergraduate dental students as dentists, and they may ask them for help in incidence of dental trauma [19]. Various studies all over the world pointed that interns or general practitioners have insufficient knowledge in treating dental trauma [20-24]. The current study aimed to assess and compare the knowledge and attitude of dental interns toward management of avulsed teeth among Egyptian and Saudi dental interns.

\section{Subjects And Methods}

This cross sectional study was conducted in Egypt and Saudi Arabia from October to December, 2019. A well-structured close ended questionnaire was constructed using my Google drive forms (https://docs.google.com/forms/d/1yqlfqcLtwG20nZ MyiaqiJRAhty-W0XBrJwLMv3LFkY8/edit). The questionnaire was sent through WhatsApp to intern students' coordinators in several dental colleges (public and private) in Egypt (Mansoura Dental College and Delta Private Dental College), and Saudi Arabia (Al-Farabi private college, King Saud Dental College, Dar El Olum private Dental College) who then sent it to their students via the internship's groups.

The questionnaire was validated and used in previous studies [25-29]. It was in English language and consists of 18 questions divided into two parts; part I about demographic data and knowledge of interns regarding the country, age, gender, months of internship experience, if they attended any educational program about management of dental trauma, and whether if they could differentiate between primary and permanent teeth. Part II is used to assess the interns' knowledge and attitude about management of avulsed teeth.

Ethical approval was obtained from the institutional research committee in Faculty of Dentistry, Mansoura University. A brief description about the purpose of the study was sent to the interns along with the questionnaires to get their consents.

\section{Statistical analysis}

Data from questionnaires was collected, organized, tabulated, coded and analyzed using SPSS version 21(IBM SPSS Statistics for Windows, Version 21.0. Armonk, NY: IBM Corp). Chi square test was used to assess percentage and frequencies of independent variables. The relation between the knowledge and the independent variables was estimated. The $\mathrm{p}$ value was considered statistical significant at $\mathrm{p} \leq 0.05$.

\section{Results}

324 dental interns out of 415(92/out of 130 Saudi and 232/ out of 285 Egyptian) with response rate $78 \%$ (70.8\% for Saudi and $81.4 \%$ for Egyptians) participated in the study and returned the completed questionnaires on the same day. There were statistically significant differences between Saudi and Egyptian dental interns regarding age $(p=0.004)$, months of internship experience $(\mathrm{p}=0.000)$, attendance of educational program related to traumatic injuries $(\mathrm{p}=0.000)$ and seeing a patient with an avulsion of a tooth $(\mathrm{p}=0.000)$ (Table 1$)$.

Table 1. Demographic Characteristics and Prior Exposure Related to Avulsion Tooth of Dental Interns.

\begin{tabular}{|c|c|c|c|}
\hline Questionnaire items & $\begin{array}{c}\text { Saudi } \\
\text { No(\%) }\end{array}$ & $\begin{array}{c}\text { Egyptian } \\
\text { No }(\%)\end{array}$ & $\begin{array}{c}\text { Total } \\
\text { No (\%) }\end{array}$ \\
\hline \multicolumn{4}{|c|}{ Gender } \\
\hline Male & $32(34.8)$ & $64(27.6)$ & $96(29.6)$ \\
\hline Female & $60(65.2)$ & $168(72.4)$ & $228(70.4)$ \\
\hline $\mathbf{P}$ & \multicolumn{3}{|c|}{0.225} \\
\hline \multicolumn{4}{|c|}{ Age groups (years) } \\
\hline Less than 25 years & $68(74)$ & $204(87.9)$ & $272(84)$ \\
\hline More than 25 years & $24(26)$ & $28(12.1)$ & $52(16)$ \\
\hline $\mathbf{P}$ & \multicolumn{3}{|c|}{0.004} \\
\hline \multicolumn{4}{|c|}{ Months of internship experience } \\
\hline$>6$ months & $68(74)$ & $216(93.1)$ & $284(87.7)$ \\
\hline$\leq 6$ months & $24(26)$ & $16(6.9)$ & $40(12.3)$ \\
\hline $\mathbf{P}$ & \multicolumn{3}{|c|}{0.000} \\
\hline \multicolumn{4}{|c|}{ Prior knowledge about management of avulsed tooth } \\
\hline Yes & $80(87)$ & $212(91.3)$ & $292(90.1)$ \\
\hline No & $12(13)$ & $20(7.8)$ & $32(9.9)$ \\
\hline $\mathbf{P}$ & \multicolumn{3}{|c|}{0.224} \\
\hline \multicolumn{4}{|c|}{ Can you differentiate between the primary and permanent teeth? } \\
\hline Yes & $92(100)$ & $232(100)$ & $324(100)$ \\
\hline No & $0(0)$ & $0(0)$ & $0(0)$ \\
\hline $\mathbf{P}$ & \multicolumn{3}{|c|}{--.---.- } \\
\hline \multicolumn{4}{|c|}{ Did you attend any educational program regarding traumatic injuries to teeth? } \\
\hline Yes & $56(60.9)$ & $76(32.8)$ & $132(40.7)$ \\
\hline No & $36(39.1)$ & $156(67.20$ & $192(59.3)$ \\
\hline $\mathbf{P}$ & \multicolumn{3}{|c|}{0.000} \\
\hline \multicolumn{4}{|c|}{ Did you ever see a patient with an avulsion of a tooth? } \\
\hline Yes & $36(39.1)$ & $44(18.9)$ & $80(24.7)$ \\
\hline No & $56(60.1)$ & $188(81.1)$ & $244(75.3)$ \\
\hline $\mathbf{P}$ & \multicolumn{3}{|c|}{0.000} \\
\hline TOTAL & $92(100)$ & $232(100)$ & $324(100)$ \\
\hline
\end{tabular}

No $(\%)=$ number and percentage, $\mathrm{p}=\mathrm{p}$ value of comparison between Saudi and Egyptian dental intern, all comparisons calculated using Chi square test and 2 -sided $\mathrm{p}$ value.

Comparison between Saudi and Egyptian dental interns indicated that, there were statistically significant differences for answering all questions related to knowledge of management of avulsion tooth $(\mathrm{p}=0.000)$ except question about what should you do when found the knocked out tooth and it is dirty $(p=0.100)$ and should primary tooth be replanted $(\mathrm{p}=0.737)$ (Table 2).

Table 2. Knowledge of dental interns related to management of avulsion tooth.

\begin{tabular}{|c|c|c|c|}
\hline Questionnaire items & $\begin{array}{c}\text { Saudi } \\
\text { No (\%) }\end{array}$ & $\begin{array}{c}\text { Egyptian } \\
\text { No (\%) }\end{array}$ & $\begin{array}{c}\text { Total } \\
\text { No (\%) }\end{array}$ \\
\hline \multicolumn{4}{|c|}{ What is avulsion of tooth? } \\
\hline $\begin{array}{c}\text { Tooth dislodgement of intact tooth out of its } \\
\text { socket, due to any trauma }\end{array}$ & $80(87)$ & $232(100)$ & $312(96.3)$ \\
\hline $\begin{array}{c}\text { Dislodgement of fractured segment of the } \\
\text { tooth due to any trauma }\end{array}$ & $12(13)$ & $0(0)$ & $12(3.7)$ \\
\hline P & \multicolumn{3}{|c|}{0.000} \\
\hline Can an avulsed permanent tooth be replanted in all cases \\
\hline Yes & $28(30.4)$ & $28(12.1)$ & $56(17.3)$ \\
\hline No & $64(69.6)$ & $204(87.9)$ & $268(82.7)$ \\
\hline P & \multicolumn{3}{|c|}{0.000} \\
\hline What is the ideal time for the re plantation of an avulsed tooth? \\
\hline 15 minutes & $20(21.8)$ & $120(51.9)$ & $140(43.2)$ \\
\hline 30 minutes & $40(43.5)$ & $54(23.3)$ & $94(29)$ \\
\hline 45 minutes & $0(0)$ & $8(3.4)$ & $8(2.5)$ \\
\hline 1 hour & $32(34.7)$ & $50(21.4)$ & $82(25.3)$ \\
\hline
\end{tabular}




\begin{tabular}{|c|c|c|c|}
\hline $\mathrm{P}$ & \multicolumn{3}{|c|}{0.000} \\
\hline \multicolumn{4}{|c|}{ You found the knocked-out tooth and it is dirty, will you........ } \\
\hline $\begin{array}{l}\text { Rinse the tooth gently under running tap water } \\
\text { for a few seconds without scrubbing it }\end{array}$ & $80(87)$ & $72(91.4)$ & $292(90.1)$ \\
\hline No need to clean the tooth because it is useless & $12(13)$ & $16(6.9)$ & $28(8.6)$ \\
\hline Clean the tooth with a toothbrush & $0(0)$ & $4(1.7)$ & $4(1.2)$ \\
\hline $\mathrm{P}$ & \multicolumn{3}{|c|}{0.100} \\
\hline \multicolumn{4}{|c|}{$\begin{array}{l}\text { What would be the best medium selected to preserve the tooth before getting profes- } \\
\text { sional care? }\end{array}$} \\
\hline Patient saliva & $12(13)$ & $111(47.9)$ & $123(38)$ \\
\hline Milk & $78(84.8)$ & $109(47)$ & $187(57.7)$ \\
\hline Saline & $2(2.2)$ & $12(5.1)$ & $14(4.3)$ \\
\hline $\mathrm{P}$ & \multicolumn{3}{|c|}{0.000} \\
\hline \multicolumn{4}{|c|}{ What is the most critical factor in the outcome of replanted tooth? } \\
\hline Extra alveolar dry time & $68(74)$ & $180(77.6)$ & $248(76.5)$ \\
\hline Storage media & $12(13)$ & $12(5.2)$ & $24(7.4)$ \\
\hline Splinting period & $0(0)$ & $24(10.4)$ & $24(7.4)$ \\
\hline Not sure & $12(13)$ & $16(6.9)$ & $28(8.6)$ \\
\hline $\mathrm{P}$ & \multicolumn{3}{|c|}{0.000} \\
\hline \multicolumn{4}{|c|}{ Type of splint used is........... } \\
\hline Flexible & $36(39.1)$ & $108(46.6)$ & $144(44.4)$ \\
\hline Rigid & $52(56.5)$ & $56(24.1)$ & $108(33.3)$ \\
\hline Anyone & $4(4.4)$ & $8(3.4)$ & $12(3.7)$ \\
\hline Not sure & $0(0)$ & $60(25.9)$ & $60(18.5)$ \\
\hline $\mathrm{P}$ & \multicolumn{3}{|c|}{0.000} \\
\hline \multicolumn{4}{|c|}{ Splinting period is for } \\
\hline 2 weeks & $40(43.4)$ & $109(47)$ & $149(46)$ \\
\hline 4 weeks & $33(35.8)$ & $48(20.7)$ & $81(25)$ \\
\hline 6 weeks & $15(16.3)$ & $16(6.9)$ & $31(9.6)$ \\
\hline Not sure & $4(4.4)$ & $59(25.4)$ & $63(19.4)$ \\
\hline $\mathrm{P}$ & \multicolumn{3}{|c|}{0.000} \\
\hline \multicolumn{4}{|c|}{ Which one has better prognosis? } \\
\hline Open apex (immature tooth) & $56(60.9)$ & $84(36.2)$ & $140(43.2)$ \\
\hline Closed apex (mature tooth) & $36(39.1)$ & $148(63.8)$ & $184(56.8)$ \\
\hline $\mathrm{P}$ & \multicolumn{3}{|c|}{0.000} \\
\hline \multicolumn{4}{|c|}{ Should primary tooth be replanted? } \\
\hline Yes & $16(17.4)$ & $36(15.6)$ & $52(16)$ \\
\hline No & $76(82.6)$ & $196(84.4)$ & $272(84)$ \\
\hline $\mathrm{P}$ & \multicolumn{3}{|c|}{0.737} \\
\hline Total & $92(100)$ & $232(100)$ & $324(100)$ \\
\hline
\end{tabular}

No $(\%)=$ number and percentage, $\mathrm{p}=\mathrm{p}$ value of comparison between Saudi and Egyptian dental intern, all comparisons calculated using $\mathrm{Chi}$ square test and 2 -sided $\mathrm{p}$ value.

Experience level of the dental interns was based on months of internship period and the more the experience, the better the response. This was statistically significant in question regarding; what should you do when found the knocked out tooth and it is dirty $(\mathrm{p}=0.026)$, type of splint used $(0.000)$, splinting period $(\mathrm{p}=0.000)$ and whether primary teeth should be replanted or not $(\mathrm{p}=0.003)$. Respondents with prior knowledge about management of avulsed tooth were better in answering the questions correctly. This was statistically significant in questions on whether avulsed tooth can be replanted in all cases $(\mathrm{p}=0.000)$, what should you do when found the knocked out tooth and it is dirty $(p=0.049)$, what is the most critical factor in the outcome of replanted tooth $(p=0.000)$ and the splinting period $(p=0.009)$. Dental interns who had not attended an educational program regarding traumatic injuries to teeth showed significant incorrect answering on splinting period $(p=0.015)$, which tooth has better prognosis $(p=0.012)$ and whether primary teeth should be replanted or not $(p=0.001)$. Dental interns who had come cross with patients with avulsion tooth showed significant better results in answering correctly on question asking about whether re plantation of an avulsed tooth can be done in all cases or not $(\mathrm{p}=0.001)$ (Table 3$)$.

Dental interns answered 4 questions sufficiently correct; Can an avulsed permanent tooth be replanted in all cases? (82.7\%), what should you do when found knocked out tooth and it is dirty? $(90.1 \%)$, what is the most critical factor in the outcome of replanted tooth? $(76.5 \%)$ and Should primary tooth be replanted? $(84 \%)$. On the other hand, they answered 4 questions insufficiently; what is the ideal time for the re-plantation of an avulsed tooth (43.2\%), Type of splint used (44.4\%), Splinting period (46\%) and which tooth has better prognosis? $(43.2 \%)$. There were no significant differences between Saudi and Egyptian dental interns who correctly answering questions related to knowledge about avulsion tooth except for 3 questions; Can an avulsed permanent tooth be replanted in all cases? $(p=0.000)$, what should you do when found knocked out tooth and it is dirty? $(\mathrm{p}=0.000)$ and which tooth has better prognosis? $(p=0.000)$. Also, there were no significant differences between male and female dental interns who answering questions correctly except for 2 questions; what should you do when found knocked out tooth and it is dirty? $(\mathrm{p}=0.013)$ and what is splinting period? $(\mathrm{p}=0.000)$ (Table 4).

Table 3. Association of selected factors with knowledge related to management of avulsion tooth among dental interns.

\begin{tabular}{|c|c|c|c|c|c|c|c|c|c|c|c|c|c|c|c|c|c|c|c|c|c|c|c|c|}
\hline \multirow{4}{*}{$\begin{array}{l}\text { Vari- } \\
\text { ables }\end{array}$} & \multicolumn{3}{|c|}{$\begin{array}{c}\text { Can an avulsed } \\
\text { permanent tooth be } \\
\text { replanted in all cases? }\end{array}$} & \multicolumn{3}{|c|}{$\begin{array}{l}\text { You found the knocked-out } \\
\text { tooth and it is dirty, will } \\
\text { you....... }\end{array}$} & \multicolumn{3}{|c|}{$\begin{array}{l}\text { What is the ideal time for } \\
\text { the re-plantation of } \\
\text { an avulsed tooth }\end{array}$} & \multicolumn{3}{|c|}{$\begin{array}{l}\text { What is the most critical } \\
\text { factor in the outcome of } \\
\text { replanted tooth? }\end{array}$} & \multicolumn{3}{|c|}{ Type of splint used } & \multicolumn{3}{|c|}{ Splinting period is for } & \multicolumn{3}{|c|}{ Which one has better prognosis? } & \multicolumn{3}{|c|}{$\begin{array}{l}\text { Should primary tooth be } \\
\text { replanted? }\end{array}$} \\
\hline & \multirow{3}{*}{$\begin{array}{c}\text { Cor- } \\
\text { rectan- } \\
\text { swer } \\
\text { No(\%) }\end{array}$} & $\begin{array}{l}\text { Incor- } \\
\text { rect }\end{array}$ & \multirow{3}{*}{$\mathbf{P}$} & \multirow{3}{*}{$\begin{array}{c}\text { Cor- } \\
\text { rectan- } \\
\text { swer } \\
\text { No(\%) }\end{array}$} & \multirow{3}{*}{$\begin{array}{l}\text { Incor- } \\
\text { rectan- } \\
\text { swer } \\
\text { No }(\%)\end{array}$} & \multirow{3}{*}{$\mathbf{P}$} & \multirow{3}{*}{$\begin{array}{c}\text { Cor- } \\
\text { rectan- } \\
\text { swer } \\
\text { No(\%) }\end{array}$} & \multirow{3}{*}{$\begin{array}{l}\text { Incor- } \\
\text { rectan- } \\
\text { swer } \\
\text { No(\%) }\end{array}$} & \multirow{3}{*}{$\mathbf{P}$} & \multirow{3}{*}{$\begin{array}{c}\text { Cor- } \\
\text { rectan- } \\
\text { swer } \\
\text { No(\%) }\end{array}$} & \multirow{3}{*}{$\begin{array}{c}\text { Incor- } \\
\text { rectan- } \\
\text { swer } \\
\text { Noo(\%) }\end{array}$} & \multirow{3}{*}{$\mathbf{P}$} & \multirow{3}{*}{$\begin{array}{c}\text { Cor- } \\
\text { rectan- } \\
\text { swer } \\
\text { No(\%) }\end{array}$} & \multirow{3}{*}{$\begin{array}{l}\text { Incor- } \\
\text { rectan- } \\
\text { swer } \\
\text { No(\%) }\end{array}$} & \multirow{3}{*}{$\mathbf{P}$} & \multirow{3}{*}{$\begin{array}{c}\text { Cor- } \\
\text { rectan- } \\
\text { swer } \\
\text { No(\%) }\end{array}$} & \multirow{3}{*}{$\begin{array}{l}\text { Incor- } \\
\text { rectan- } \\
\text { swer } \\
\text { No(\%) }\end{array}$} & \multirow{3}{*}{$\mathrm{P}$} & $\begin{array}{l}\begin{array}{c}\text { Correct } \\
\text { answert }\end{array} \\
\end{array}$ & $\begin{array}{c}\text { Incorrect } \\
\text { answer }\end{array}$ & \multirow{3}{*}{$\mathrm{P}$} & Correct & $\begin{array}{c}\text { Incor- } \\
\text { rect }\end{array}$ & \multirow{3}{*}{$\mathbf{P}$} \\
\hline & & answer & & & & & & & & & & & & & & & & & No(\%) & $\mathrm{No}(\%)$ & & answer & answer & \\
\hline & & $\mathrm{No}(\%)$ & & & & & & & & & & & & & & & & & & & & $\mathrm{No}(\%)$ & No(\%) & \\
\hline \multicolumn{25}{|c|}{ Months of internship - Experience } \\
\hline $\begin{array}{c}>6 \\
\text { months }\end{array}$ & $\begin{array}{c}232 \\
(86.6)\end{array}$ & $\begin{array}{c}52 \\
(13.4)\end{array}$ & 0.1 & $\begin{array}{c}252 \\
(88.7) \\
\end{array}$ & $\begin{array}{c}32 \\
-13.7\end{array}$ & 002 & $\begin{array}{l}128 \\
(45)\end{array}$ & $\begin{array}{l}156 \\
(55)\end{array}$ & D & \begin{tabular}{c|}
220 \\
$(77.5)$
\end{tabular} & $\begin{array}{c}64 \\
(22.5)\end{array}$ & D & $\begin{array}{l}112 \\
(39.4)\end{array}$ & $\begin{array}{l}172 \\
(60.6) \\
\end{array}$ & D & $\begin{array}{c}117 \\
(41.2)\end{array}$ & $\begin{array}{c}167 \\
(58.8)\end{array}$ & 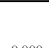 & $\begin{array}{c}124 \\
(43.7)\end{array}$ & $\begin{array}{l}160 \\
(56.3)\end{array}$ & 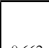 & $\begin{array}{l}232 \\
(81.7)\end{array}$ & $\begin{array}{c}52 \\
(18.3)\end{array}$ & 0000 \\
\hline $\begin{array}{c}\leq 6 \\
\text { months }\end{array}$ & $\begin{array}{c}36 \\
(90)\end{array}$ & $\begin{array}{c}4 \\
(10)\end{array}$ & 0.14 .3 & $\begin{array}{c}40 \\
(100)\end{array}$ & $\begin{array}{c}0 \\
(0)\end{array}$ & 0.026 & $\begin{array}{l}12 \\
(30)\end{array}$ & $\begin{array}{c}28 \\
(70)\end{array}$ & 0.072 & $\begin{array}{c}28 \\
(70)\end{array}$ & $\begin{array}{l}12 \\
(30)\end{array}$ & 0.298 & $\begin{array}{c}32 \\
(80)\end{array}$ & $\begin{array}{c}8 \\
(20)\end{array}$ & 0.000 & $\begin{array}{c}32 \\
(80)\end{array}$ & $\begin{array}{c}8 \\
(20)\end{array}$ & 0.000 & $\begin{array}{l}16 \\
(40)\end{array}$ & $\begin{array}{c}24 \\
(60)\end{array}$ & 0.662 & $\begin{array}{c}40 \\
(100)\end{array}$ & $\begin{array}{l}0 \\
(0)\end{array}$ & 0.005 \\
\hline & & & & & & & & & & Prior & knowledgc & about $\mathrm{m}$ & agement of & avulsed too & & & & & & & & & & \\
\hline Yes & $\begin{array}{c}252 \\
(86.3)\end{array}$ & $\begin{array}{c}40 \\
(13.7)\end{array}$ & 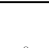 & $\begin{array}{l}260 \\
{ }_{(89)}\end{array}$ & $\begin{array}{c}32 \\
\text { (11) }\end{array}$ & 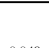 & $\begin{array}{c}124 \\
(42.5)\end{array}$ & $\begin{array}{l}168 \\
(57.5)\end{array}$ & $04=$ & $\begin{array}{c}236 \\
(80.8)\end{array}$ & $\begin{array}{c}56 \\
(19.2)\end{array}$ & 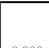 & $\begin{array}{c}128 \\
(43.8)\end{array}$ & $\begin{array}{l}164 \\
(56.2)\end{array}$ & $x=$ & $\begin{array}{c}141 \\
(48.3)\end{array}$ & $\begin{array}{c}151 \\
(51.7)\end{array}$ & 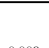 & $\begin{array}{c}124 \\
(42.5)\end{array}$ & $\begin{array}{c}168 \\
(57.5)\end{array}$ & 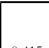 & $\begin{array}{c}244 \\
(83.6)\end{array}$ & $\begin{array}{c}48 \\
(16.4)\end{array}$ & $x_{-1}$ \\
\hline No & $\begin{array}{l}16 \\
(50)\end{array}$ & $\begin{array}{l}16 \\
(50)\end{array}$ & 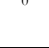 & $\begin{array}{c}32 \\
(100)\end{array}$ & $\begin{array}{c}0 \\
\text { (0) }\end{array}$ & 0.0479 & $\begin{array}{l}16 \\
(50)\end{array}$ & $\begin{array}{l}16 \\
(50)\end{array}$ & 0.413 & \begin{tabular}{|c|}
12 \\
$(37.5)$ \\
\end{tabular} & $\begin{array}{c}20 \\
(62.5)\end{array}$ & 0.000 & $\begin{array}{l}16 \\
(50)\end{array}$ & $\begin{array}{l}16 \\
(50)\end{array}$ & 10.500 & $\begin{array}{c}8 \\
(25)\end{array}$ & $\begin{array}{l}24 \\
(75) \\
\end{array}$ & 0.009 & $\begin{array}{l}16 \\
(50)\end{array}$ & $\begin{array}{l}16 \\
(50)\end{array}$ & 0.413 & $\begin{array}{c}28 \\
(87.5)\end{array}$ & $\begin{array}{c}4 \\
(12.5)\end{array}$ & 0.503 \\
\hline & & & & & & & & & & rou attend a & yy cducatio & nal progr & $n$ regarding & raumatic inj & uries to & ecth? & & & & & & & & \\
\hline Yes & $\begin{array}{c}112 \\
(84.8)\end{array}$ & $\begin{array}{c}20 \\
(15.2)\end{array}$ & 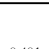 & $\begin{array}{c}120 \\
(90.1)\end{array}$ & $\begin{array}{l}12 \\
(9.9)\end{array}$ & 060 & $\begin{array}{c}52 \\
(39.4)\end{array}$ & $\begin{array}{c}80 \\
(60.1)\end{array}$ & 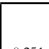 & \begin{tabular}{c|}
100 \\
$(75.8)$
\end{tabular} & $\begin{array}{c}32 \\
(24.2)\end{array}$ & 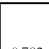 & $\begin{array}{c}48 \\
(36.4)\end{array}$ & \begin{tabular}{|c|}
84 \\
$(63.6)$ \\
\end{tabular} & 1 & $\begin{array}{c}68 \\
(51.5)\end{array}$ & $\begin{array}{c}64 \\
(48.5)\end{array}$ & 00 & $\begin{array}{l}68 \\
(51.5)\end{array}$ & $\begin{array}{c}64 \\
(48.5)\end{array}$ & 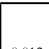 & $\begin{array}{l}100 \\
(75.8)\end{array}$ & $\begin{array}{c}32 \\
(24.2)\end{array}$ & 0 \\
\hline No & $\begin{array}{c}156 \\
(81.3)\end{array}$ & $\begin{array}{c}36 \\
(18.7)\end{array}$ & 0.401 & $\begin{array}{c}172 \\
(89.6)\end{array}$ & $\begin{array}{c}20 \\
(10.4)\end{array}$ & 0.695 & $\begin{array}{c}88 \\
(45.8)\end{array}$ & $\begin{array}{c}104 \\
(54.2)\end{array}$ & 0.251 & \begin{tabular}{c|}
148 \\
$(77.1)$
\end{tabular} & $\begin{array}{c}44 \\
(22.9)\end{array}$ & 0.782 & $\begin{array}{c}96 \\
(50)\end{array}$ & $\begin{array}{c}96 \\
(50)\end{array}$ & 0.015 & $\begin{array}{c}81 \\
(42.2)\end{array}$ & $\begin{array}{c}111 \\
(57.8)\end{array}$ & 0.098 & $\begin{array}{c}72 \\
(37.5)\end{array}$ & $\begin{array}{c}120 \\
(62.5)\end{array}$ & 0.012 & $\begin{array}{c}172 \\
(89.06)\end{array}$ & $\begin{array}{c}20 \\
(10.4)\end{array}$ & 0.001 \\
\hline No & $\begin{array}{l}56 \\
(70)\end{array}$ & $\begin{array}{l}24 \\
(30)\end{array}$ & & $\begin{array}{l}68 \\
(85)\end{array}$ & $\begin{array}{l}12 \\
(15)\end{array}$ & & $\begin{array}{c}34 \\
(42.5)\end{array}$ & $\begin{array}{l}46 \\
(57.5)\end{array}$ & & $\begin{array}{l}64 \\
640) \\
(80)\end{array}$ & $\begin{array}{l}16 \\
(20)\end{array}$ & 0.401 & $\begin{array}{l}36 \\
(45)\end{array}$ & $\begin{array}{l}44 \\
(55)\end{array}$ & & $\begin{array}{l}44 \\
(55)\end{array}$ & $\begin{array}{l}36 \\
(45)\end{array}$ & 0.003 & $\begin{array}{l}36 \\
(45)\end{array}$ & $\begin{array}{l}44 \\
(55)\end{array}$ & 0.11 & $\begin{array}{l}68 \\
(85)\end{array}$ & $\begin{array}{l}12 \\
(15)\end{array}$ & $0.10 \%$ \\
\hline
\end{tabular}

No $(\%)=$ number and percentage, $\mathrm{p}=$ association between variables and it was calculated by $\mathrm{Chi}$ square linear association. 
Table 4. Comparison between knowledge related to management of avulsion tooth between Saudi and Egyptian \& male and female dental interns.

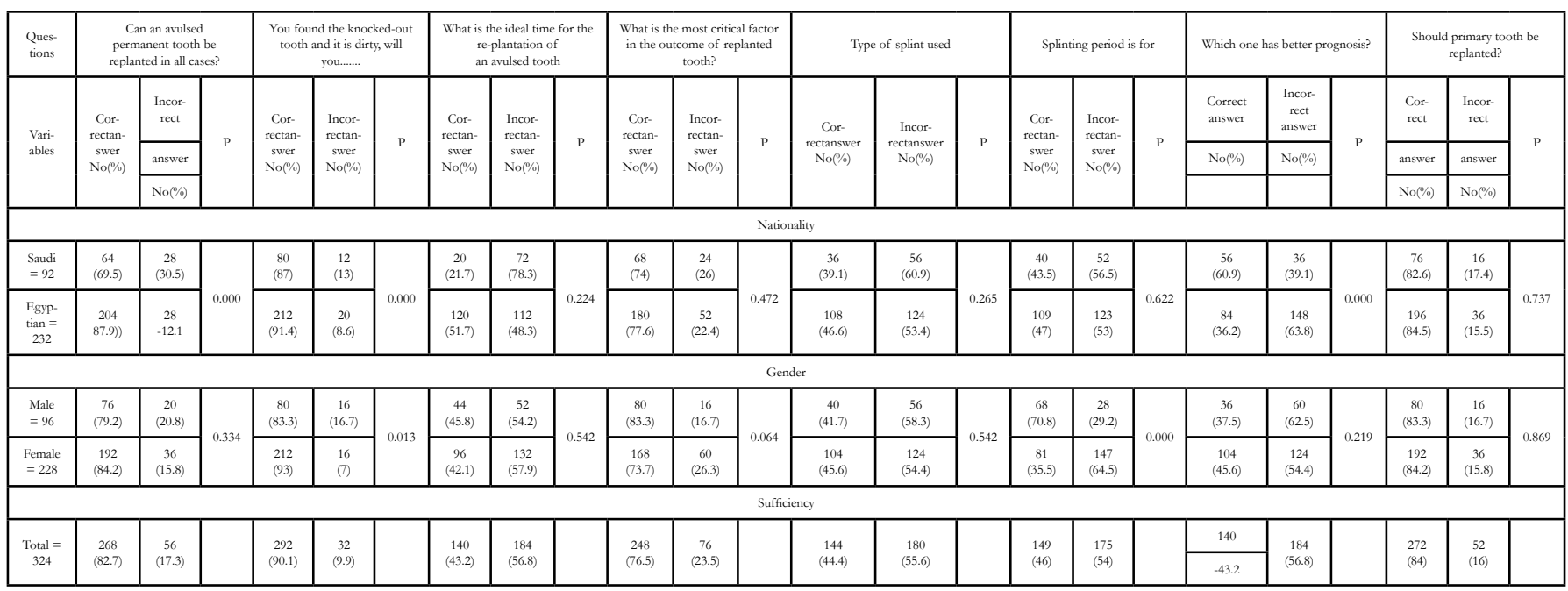

No $(\%)=$ number and percentage, $\mathrm{p}=\mathrm{p}$ value calculated by Chi square test.

\section{Discussion}

After conducting comprehensive databases searching (Google Scholar, PubMed, and Medline), there were no available data about knowledge of tooth avulsion management among dental interns or dental practitioners in Egypt or data about comparison of the knowledge level between Egyptian and Saudian dental interns. So this study was conducted to explore and compare the knowledge level between them.

The finding of the present study revealed that, the majority of our participants were females $(70.4 \%)$, and this could be attributed to the general behavior of females as they are usually more active and interactive than males, this finding was matched with that of Azmi and Awooda [30].

It was found that most of our participants had responded correctly to most of the questions, except that about prognosis of mature or immature teeth after replantation and the suitable type of splint, and this could be explained by the fresh knowledge that they acquired during their undergraduate study. These findings were agreed with Azmi and Awooda [30] and Alaslami et al., [31]. On the other hand, Al-Shamri et al., [32] reported low knowledge level regarding dental trauma management among their participants in Saudi Arabia.

It was found that majority of Egyptian and Saudi dental interns did not attend any educational programs related to management of dental trauma, and this was matched with Al-Shamiri et al [32], whofound a significant shortage in attending courses about traumatic dental injury. Aljazairy et al., [33] reported that, no significant difference in knowledge between those who had and had not attended a continuous dental education program. Thisfindingwas not consistent with several previous studies [34-37] which reported that dentists had higher knowledge scores, had more thorough and better knowledge of dental trauma management and had more confidence in managing these patients after attended post graduate dental trauma courses.
Regarding the knowledge about management of avulsed teeth, $96.3 \%$ of our participants defined tooth avulsion correctly and this was matched with Limbu et al [38], however, $7 \%$ only of their respondents knew that not all avulsed permanent teeth can be replanted in comparison to $82.7 \%$ of our respondents.

The duration of avulsed tooth outside the socket is one of the important factors to preserve its vitality after replantation. One of the previous studies reported that teeth replanted within 5 minutes had the best prognosis [39] while other studies suggested that 20 to 30 minutes is the maximum limit $[13,14]$. About $43.2 \%$ of our respondents cleared that 15 minutes is the suitable period to replant avulsed tooth, also $90.1 \%$ of our participants cleared that washing avulsed tooth under running water without scrubbing it is the ideal action after TDIs, these findings were agreed with Limbu's [38].

Several studies demonstrated that patient's saliva [27], saline [23], or milk [40-42] are the preferable or recommended storage medium. The majority ofour respondents confirmed that milk is the suitable media for reserving avulsed tooth, this was matched with those of (IADT) [9], (AAPD) [8] guidelines and Al-Shamiri et al results [32].

About $76.5 \%$ of our respondents cleared that, the most critical factor in replantation success is the extra-alveolar drying time and this was agreed with several studies [43-45].

About $44.4 \%$ of our participants seen that flexible splint is the correct option and $46 \%$ of them mentioned that 2 weeks is the ideal duration for splinting. There was a controversy about that finding among several studies, as $\mathrm{Hu}$ et al[36] reported $59.1 \%$ agreement among their respondents that a flexible splint should be used for fixation of avulsed teeth for 2 weeks. AlsoWestphalen et al[43] reported that, $73 \%$ of their respondents selected flexible splint and $64 \%$ of them reported splinting duration of 15 days or more. On the other hand, Zhao et al[45]reported that,49\% of their respondents suggested rigid splint and $40.6 \%$ suggested splinting duration for 30 days while $10.2 \%$ only suggested splinting for 2 weeks. 
Cvek et al., [46] reported that teeth with incomplete or open apices if replanted within 60 minutes of avulsion will have higher chances of recovery by pulp revascularizationand periodontal healing occurs more frequently faster than in teeth with closed apex. About $56.8 \%$ of our respondents realized that immature tooth has better prognosis than mature tooth and this was better than the results obtained by Limbu et al., [38] where only $26 \%$ of their respondents got the correct response.

Zamon and Kenny [47] expected that replantation of avulsed deciduous tooth can cause deflection, hypoplastic and morphologic abnormalities to the crown of successors, and it may also form a dental abscess or undergo ankylosis. $84 \%$ of our participants deduced that, primary teeth should not be replanted.This finding matched with the guidelines of AAPD and IADT, and the results of Limbu et al., [38] also revealed 86\% agreement with our results.

Saudi and Egyptian dental interns' knowledge about management of avulsed teeth are somewhat near each other, there were no statistical significant differences between them regarding most of the questions while the Egyptian were answered correctly two questions significantly higher than Saudi and the vice versa for one question was observed (Table 4). Our results confirm and support the results obtained by Alaslami et al., [31] as Saudi dental interns replied correctly to most questions and had enough level of knowledge. However, Al-Shamiri et al, 2015 [32] concluded that, dental students had insufficient knowledge concerning dental trauma management and they recommended a variety of educational methods such as problem-based learning and powering the curriculum concerning those topicsto improve the knowledge of dental students. Also, AlJazairy et al., [33] cleared that, their participants were found to have a moderate level of knowledge about tooth avulsion management. In Egypt there was no previous data about interns'or dentists' knowledge regarding management of avulsed teeth to compare with it, but our results revealed good knowledge level of Egyptian dental interns in relation to that of Saudi dental interns.

\section{Conclusion}

According tothe results of this study and in relation to the previous results, the Saudi and Egyptian dental interns had sufficient level of knowledge about most items regardingtooth avulsion management. Also, the present results indicated that, the dental interns should be motivated for attending more educational program regarding traumatic injuries to teeth.

\section{Study Limitations}

It has been reported that questionnaire based cross-sectional studies are prone to limitation and bias. The results of self-reporting surveys may not necessary fully reflect student's real knowledge and daily professional practice.

\section{References}

[1]. American Academy on Pediatric Dentistry Council on Clinical Affairs. Guideline on management of acute dental trauma.(2008 2009) PediatrDent.30(7 Suppl): 175183.

[2]. Malmgren B, Andreasen J, Flores M, Robertson A, DiAngelis A. Guidelines for the management of traumatic dental injuries: 3 . Injuries in the primary dentition. Pediatr Dent.2016;38:377 385 .

[3]. Zaleckiene V, Peciuliene V, Brukiene V, Drukteinis S. Traumatic dental injuries: etiology, prevalence and possible outcomes. Stomatologija. 2014;16(1):7-14.Pubmed PMID: 24824054

[4]. Mohandas U, Chandan GD. Knowledge, attitude and practice in emergency management of dental injury among physical education teachers: a survey in Bangalore urban schools. J Indian Soc Pedod Prev Dent. 2009 Oct-Dec;27(4):242-8.Pubmed PMID: 19915276.

[5]. Fujita Y, Shiono Y, Maki K. Knowledge of emergency management of avulsed tooth among Japanese dental students. BMC Oral Health. $2014 \mathrm{Apr}$ 8;14:34.Pubmed PMID: 24712491.

[6]. Andersson L, Andreasen JO, Day P, Heithersay G, Trope M, Diangelis AJ, et al. International Association of Dental Traumatology. International Association of Dental Traumatology guidelines for the management of traumatic dental injuries: 2. Avulsion of permanent teeth. Dent Traumatol. 2012 Apr;28(2):88-96. Pubmed PMID: 22409417.

[7]. Andreasen JO. External root resorption: its implication in dental traumatology, paedodontics, periodontics, orthodontics and endodontics. Int Endod J. 1985 Apr;18(2):109-18.Pubmed PMID: 2860072.

[8]. Trope M. Clinical management of the avulsed tooth: present strategies and future directions. Dent Traumatol. 2002 Feb;18(1):1-11.Pubmed PMID: 11841460.

[9]. Andreasen JO, Borum MK, Jacobsen HL, Andreasen FM. Replantation of 400 avulsed permanent incisors. 4. Factors related to periodontal ligament healing. Endod Dent Traumatol. 1995 Apr;11(2):76-89.Pubmed PMID: 7641622.

[10]. Barrett EJ, Kenny DJ. Survival of avulsed permanent maxillary incisors in children following delayed replantation. Endod Dent Traumatol. 1997 Dec;13(6):269-75.Pubmed PMID: 9558508.

[11]. McDonald N, Strassler HE. Evaluation for tooth stabilization and treatment of traumatized teeth. Dent Clin North Am. 1999 Jan 1;43(1):135-49.

[12]. Donaldson M, Kinirons MJ. Factors affecting the time of onset of resorption in avulsed and replanted incisor teeth in children. Dent Traumatol. 2001 Oct;17(5):205-9.Pubmed PMID: 11678538.

[13]. Sigalas E, Regan JD, Kramer PR, Witherspoon DE, Opperman LA. Survival of human periodontal ligament cells in media proposed for transport of avulsed teeth. Dent Traumatol. 2004 Feb;20(1):21-8.Pubmed PMID: 14998411.

[14]. Chappuis V, von Arx T. Replantation of 45 avulsed permanent teeth: a 1-year follow-up study. Dent Traumatol. 2005 Oct;21(5):289-96.Pubmed PMID: 16149925.

[15]. Al-Obaida M. Knowledge and management of traumatic dental injuries in a group of Saudi primary schools teachers. Dent Traumatol. 2010 Aug;26(4):338-41.Pubmed PMID: 20662887.

[16]. Al-Majed I, Murray JJ, Maguire A. Prevalence of dental trauma in 5-6and 12-14-year-old boys in Riyadh, Saudi Arabia. Dent Traumatol. 2001 Aug;17(4):153-8.Pubmed PMID: 11585140.

[17]. Abdellatif AM, Hegazy SA. Knowledge of emergency management of avulsed teeth among a sample of Egyptian parents. J Adva Res. $2011 \mathrm{Apr}$ $1 ; 2(2): 157-62$.

[18]. El-Kalla IH, Shalan HM, Bakr RA. Impact of dental trauma on quality of life among 11-14 years schoolchildren. Contemp Clin Dent. 2017 Oct; $8(4): 538-544$.

[19]. Mustafa M. Awareness about management of tooth avulsion among general dental practitioners: A questionnaire based study. J. Orthod. 2017;3(1):2.

[20]. Kostopoulou MN, Duggal MS. A study into dentists' knowledge of the treatment of traumatic injuries to young permanent incisors. Int J Paediatr Dent. 2005 Jan;15(1):10-9.Pubmed PMID: 15663440.

[21]. Re D, Augusti D, Paglia G, Augusti G, Cotti E. Treatment of traumatic dental injuries: evaluation of knowledge among Italian dentists. Eur J Paediatr Dent. 2014 Mar;15(1):23-8.Pubmed PMID: 24745588.

[22]. Ravikumar D, Jeevanandan G, Subramanian EM. Evaluation of knowledge among general dentists in treatment of traumatic injuries in primary teeth: A cross-sectional questionnaire study. Eur. J. Dent. 2017 Apr;11(2):232-237.

[23]. Buldur B, Kapdan A. Factors associated with knowledge and attitude of management of traumatic dental injuries: A cross-sectional study among Turkish dentists. Pesqui. Bras. Odontopediatria Clín. Integr. 2018 Feb 26;18(1):1-9.

[24]. Zaleckienė V, Pečiulienė V, Brukienė V, Jakaitienė A, Aleksejūnienė J, Zaleckas L. Knowledge about traumatic dental injuries in the permanent dentition: A survey of Lithuanian dentists. Dent Traumatol. 2018 Apr;34(2):100106.Pubmed PMID: 29450968.

[25]. Holan G, Shmueli Y. Knowledge of physicians in hospital emergency rooms in Israel on their role in cases of avulsion of permanent incisors. Int J Paediatr Dent. 2003 Jan;13(1):13-19.Pubmed PMID: 12542619.

[26]. Abu-Dawoud M, Al-Enezi B, Andersson L. Knowledge of emergency management of avulsed teeth among young physicians and dentists. Dent Traumatol. 2007 Dec;23(6):348-55.Pubmed PMID: 17991234. 
[27]. Alastair NS, Heather KA, Tony MC.Professional Knowledge of method for emergency management of avulsed teeth. Dent Traumatol.2008;24:325-329.

[28]. Subhashraj K. Awareness of management of dental trauma among medical professionals in Pondicherry, India. Dent Traumatol. 2009 Feb;25(1):92-4. Pubmed PMID: 19208018

[29]. Upadhyay S, Rokaya D, Upadhayaya C. Knowledge of emergency management of avulsed teeth among general dentists in Kathmandu. Kathmandu Univ Med J (KUMJ). 2012 Apr-Jun;38(2):37-40.Pubmed PMID: 23132473.

[30]. Azmi MA, Awooda EM.Knowledge of emergency management of avulsed tooth among undergraduate preclinical and clinical dental students: Questionnaire-based study. J Dent Res Rev. 2016;3:140-143.

[31]. Alaslami RA, Elshamy FMM, Maamar EM, Ghazwani YH. Awareness about Management of Tooth Avulsion among Dentists in Jazan, Saudi Arabia. Open Access Maced J Med Sci. 2018 Sep 21;6(9):1712-1715.Pubmed PMID: 30337996.https://pubmed.ncbi.nlm.nih.gov/30337996/

[32]. Al-Shamiri HM, Alaizari NA, Al-Maweri SA, Tarakji B. Knowledge and attitude of dental trauma among dental students in Saudi Arabia. Eur J Dent. 2015 Oct;9(4):518-522.

[33]. AlJazairy YH, Halawany HS, AlMaflehi N, Alhussainan NS, Abraham NB, Jacob V. Knowledge about permanent tooth avulsion and its management among dentists in Riyadh, Saudi Arabia. BMC Oral Health. 2015 Nov 2;15(1):135.Pubmed PMID: 26527540

[34]. Hamilton FA, Hill FJ, Holloway PJ. An investigation of dento-alveolar trauma and its treatment in an adolescent population. Part 2: Dentists' knowledge of management methods and their perceptions of barriers to providing care. Br Dent J. 1997 Feb 22;182(4):129-33.Pubmed PMID: 9061998.

[35]. Jackson NG, Waterhouse PJ, Maguire A. Management of dental trauma in primary care: a postal survey of general dental practitioners. Br Dent J. 2005 Mar 12;198(5):293-7.Pubmed PMID: 15870756.

[36]. Kostopoulou MN, Duggal MS. A study into dentists' knowledge of the treatment of traumatic injuries to young permanent incisors. Int J Paediatr Dent. 2005 Jan;15(1):10-19. PMID: 15663440.

[37]. Hu LW, Prisco CR, Bombana AC. Knowledge of Brazilian general dentists and endodontists about the emergency management of dento-alveolar trau- ma. Dent Traumatol. 2006 Jun;22(3):113-117. PMID: 16643284.

[38]. Limbu S, Dikshit P, Bhagat T, Mehata S. Knowledge of dental interns towards emergency management of avulsed tooth in dental colleges in Nepal. J Nepal Health Res Counc. 2014 Jan;12(26):1-7.Pubmed PMID: 25574976.

[39]. Al-Jundi SH, Al-Waeili H, Khairalah K. Knowledge and attitude of Jordanian school health teachers regarding emergency management of dental trauma. Dent Traumatol.2005; 21(4):183- 187.

[40]. Cohenca N, Forrest JL, Rotstein I. Knowledge of oral health professionals of treatment of avulsed teeth. Dent Traumatol. 2006 Dec;22(6):296-301. Pubmed PMID: 17073920.

[41]. Yeng T, Parashos P. An investigation into dentists' management methods of dental trauma to maxillary permanent incisors in Victoria, Australia. Dent Traumatol. 2008 Aug;24(4):443-8.Pubmed PMID: 18721345.

[42]. de Vasconcellos LG, Brentel AS, Vanderlei AD, de Vasconcellos LM, Valera MC, de Araújo MA. Knowledge of general dentists in the current guidelines for emergency treatment of avulsed teeth and dental trauma prevention. Dent Traumatol. 2009 Dec;25(6):578-83.Pubmed PMID: 19788428.

[43]. Westphalen VP, Martins WD, Deonizio MD, da Silva Neto UX, da Cunha $\mathrm{CB}$, Fariniuk LF. Knowledge of general practitioners dentists about the emergency management of dental avulsion in Curitiba, Brazil. Dent Traumatol. 2007 Feb;23(1):6-8.Pubmed PMID: 17227373.

[44]. Andreason JOA. Avulsions. In: Text book and Atlas of traumatic injuries to the teeth. 4th edn. Blackwell and Munksgaad.2007; 444-480.

[45]. Zhao Y, Gong Y. Knowledge of emergency management of avulsed teeth: a survey of dentists in Beijing, China. Dent Traumatol. 2010 Jun;26(3):281-4. Pubmed PMID: 20572844.

[46]. Cvek M, Cleaton-Jones P, Austin J, Lownie J, Kling M, Fatti P. Pulp revascularization in reimplanted immature monkey incisors--predictability and the effect of antibiotic systemic prophylaxis. Endod Dent Traumatol. 1990 Aug;6(4):157-69.Pubmed PMID: 1723381.

[47]. Zamon EL, Kenny DJ. Replantation of avulsed primary incisors: a risk benefit assessment. J Can Dent Assoc. 2001 Jul-Aug;67(7):386-391.Pubmed PMID: 11468096 